\title{
UNIVERSITYOF
}

FORWARD

THINKING

WESTMINSTER用

WestminsterResearch

http://www.westminster.ac.uk/westminsterresearch

\section{A Novel Pressure Sensing Circuit for Non-invasive RF/Microwave Blood Glucose Sensors}

Turgul, V. and Kale, I.

This is a copy of the author's accepted version of a paper subsequently published in the proceedings of the IEEE 16th Mediterranean Microwave Symposium (MMS2016), Abu Dhabi 14 to16 Nov, 2016, IEEE.

It is available online at:

https://dx.doi.org/10.1109/MMS.2016.7803818

(C) 2016 IEEE . Personal use of this material is permitted. Permission from IEEE must be obtained for all other uses, in any current or future media, including reprinting/republishing this material for advertising or promotional purposes, creating new collective works, for resale or redistribution to servers or lists, or reuse of any copyrighted component of this work in other works.

The WestminsterResearch online digital archive at the University of Westminster aims to make the research output of the University available to a wider audience. Copyright and Moral Rights remain with the authors and/or copyright owners.

Whilst further distribution of specific materials from within this archive is forbidden, you may freely distribute the URL of WestminsterResearch: ((http://westminsterresearch.wmin.ac.uk/)).

In case of abuse or copyright appearing without permission e-mail repository@westminster.ac.uk 


\title{
A Novel Pressure Sensing Circuit for Non-invasive RF/Microwave Blood Glucose Sensors
}

\author{
Volkan Turgul and Izzet Kale \\ Applied DSP and VLSI Research Group (ADVRG), Department of Engineering \\ Faculty of Science and Technology, University of Westminster \\ London, W1W 6UW, United Kingdom \\ E-mail: volkan.turgul@my.westminster.ac.uk, kalei@westminster.ac.uk
}

\begin{abstract}
A novel pressure sensing circuit for non-invasive $\mathrm{RF} / \mathrm{microwave}$ blood glucose sensors is presented in this paper. RF sensors are of interest to researchers for measuring blood glucose levels non-invasively. For the measurements, the finger is a popular site that has a good amount of blood supply. When a finger is placed on top of the RF sensor, the electromagnetic fields radiating from the sensor interact with the blood in the finger and the resulting sensor response depends on the permittivity of the blood. The varying glucose level in the blood results in a permittivity change causing a shift in the sensor's response. Therefore, by observing the sensor's frequency response it may be possible to predict the blood glucose level. However, there are two crucial points in taking and subsequently predicting the blood glucose level. These points are; the position of the finger on the sensor and the pressure applied onto the sensor. A variation in the glucose level causes a very small frequency shift. However, finger positioning and applying inconsistent pressure have more pronounced effect on the sensor response. For this reason, it may not be possible to take a correct reading if these effects are not considered carefully. Two novel pressure sensing circuits are proposed and presented in this paper to accurately monitor the pressure applied.
\end{abstract}

Keywords-non-invasive; glucose; sensing; RF; microwave; pressure; sensor; permittivity

\section{INTRODUCTION}

Diabetes Mellitus is affecting over 400 million people worldwide, a well-known disease associated with high Blood Glucose Levels (BGL) [1]. This arises from the insufficient production of the insulin hormone or its ineffectiveness in the patients' body. In this case, the BGL can be controlled through medication or by administering insulin externally [2]. However, the BGL must be known prior to taking action. Conventional methods require a drop of blood to be taken from the patient, usually from a pricked fingertip, that is applied onto a test strip, which is then inserted into the measurement device for a BGL reading. Some diabetes patients may need up to 10 measurements a day; hence, the process gets uncomfortable. For this reason, researchers have been investigating various methods where the use of Radio Frequency (RF)/microwave sensors is one of them for measuring the BGL non-invasively [3]. The measurement principle lies in the detection of the permittivity of the measured media that is placed on top of the sensor. Any variation in the dielectric properties of the media will induce a change in the sensor's frequency response and the BGL can be estimated by observing this. This response is a function of multiple factors, such as; the electrical properties of biological tissue layers, constituents found in the blood and the position of the sample (finger) on the sensor [4]. Another important factor is the pressure exerted onto the sensor by the finger. These factors are explained in the following sections.

\section{BACKGROUND THEORY}

When a sample is placed on top of a transmission line, the fringing fields propagate through the sample, which acts as a superstrate as shown in Fig.1. ( $\varepsilon$ denotes the dielectric constant).

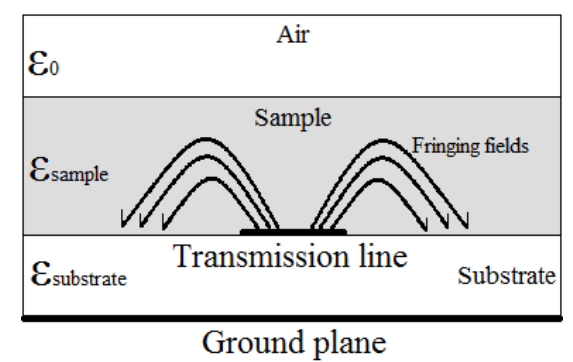

Fig.1. Interaction of fringing fields with the sample

In the case of using a fingertip as the sample, the fringing fields exist in several biological tissue layers. These layers are; skin, fat, blood and bone. The penetration depth depends on the frequency of operation of the sensor and in a good sensor design, penetration beyond the blood layer should be as limited as possible. In any case, to measure the BGL the fields need to penetrate the skin and the fat layers. The blood is found in a complex network of capillaries within the subcutaneous tissues beneath the fat layer. The BGL information is contained in the response resulting from this layer. Any response from other layers is not of interest, which is why the penetration depth should be limited to the blood layer. The 4-layer fingertip model was realized in CST Microwave Studio (MWS) electromagnetic simulation software to validate the theory. The model is shown in Fig.2 with the permittivity and thickness values of the layers. 


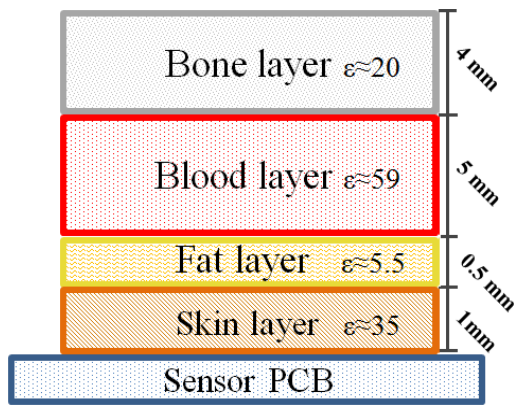

Fig. 2. The 4-layer fingertip model used for simulations

The complex permittivity of the layers was calculated using the Cole-Cole relaxation model with the parameters found in the open literature [5], [6].

\section{THE DESIGNED RF SENSOR}

A compact non-invasive RF glucose sensor was designed, fabricated and used in this study [7]. The designed sensor is based on a one-port microwave resonator, which acts as an antenna used for near-field radiation. The resonance frequency of the unloaded sensor is $4.8 \mathrm{GHz}$. When the fingertip is placed on top of the sensor, the resonance frequency shifts down to about $3.25 \mathrm{GHz}$ depending on the pressure applied. The sensor was fabricated on a Rogers RO3006 laminate with a thickness of $32 \mathrm{mil}$ and a dielectric constant of 6.5. The sensor has a main sensing area that resembles an interdigital capacitor for achieving high capacitance to maximize the electric field for increased sensitivity to permittivity change. The $50 \Omega$ feed line is located at the back of the substrate as a coplanar waveguide and is connected to the sensor through a plated via hole. This is to ensure that the finger does not come into contact with the feed line, possibly altering the sensor response. The aim was to design a compact sensor so that the whole structure is covered with the fingertip; therefore, the fringing fields are contained within the fingertip as much as possible. The layout of the designed sensor is shown in Fig. 3.

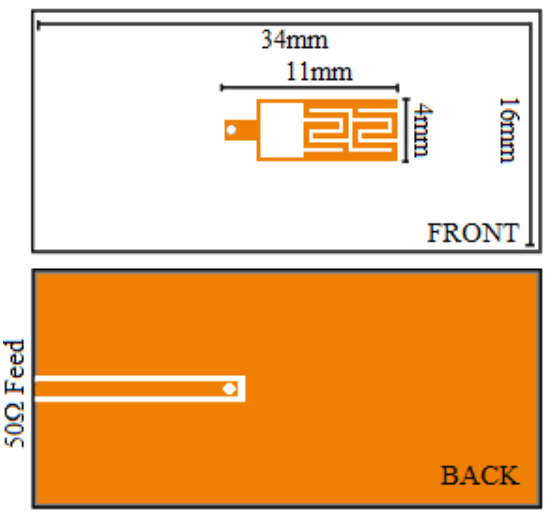

Fig. 3. Layout of the desgined sensor

The sensor was simulated in CST MWS and measured in air for the unloaded response using a VNA. It was also simulated with the 4-layer fingertip model and measured with an actual fingertip. The simulated and measured responses are shown in Fig. 4.

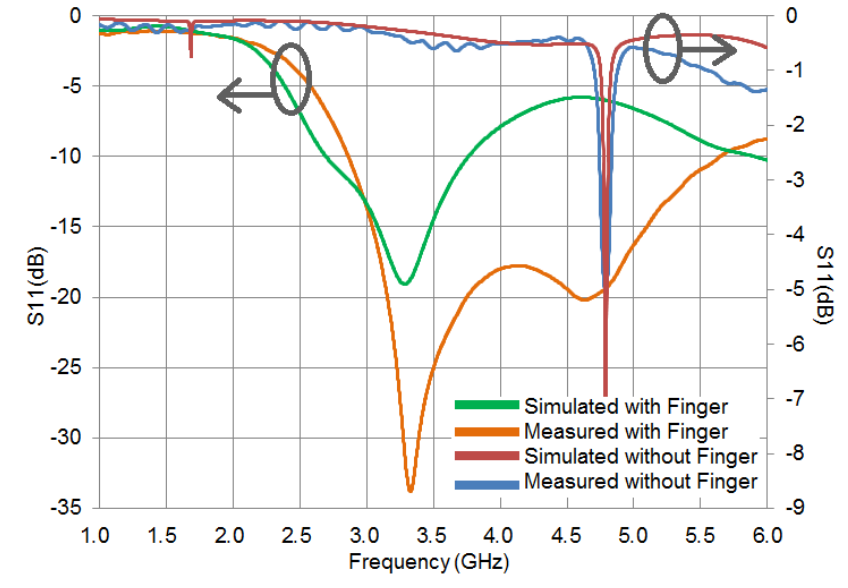

Fig. 4. Measured and simulated return loss (S11) of the sensor with and without finger

There is a good agreement between both when the sensor is unloaded. The simulated response with the fingertip model and the measured response with an actual finger show a resonant peak at the same frequency; however, it can be observed that the Q-factor of the sensor is lower in the measured case leading to a wideband resonance. This is due to the simplified nature of the 4-layer fingertip model used in CST MWS and a more accurate model is difficult to achieve due to the complex structure of the tissues.

\section{The Finger Position And Pressure Problems}

The importance of the positioning of the finger on the sensor and the pressure applied as well as their effects are explained in this section.

\section{A. Finger Position on the Sensor}

The fingertip should cover the whole sensing area and should be positioned in the same place every time for a repeatable measurement. Otherwise, different proportions of fringing fields may exist in air and tissue layers with unknown fractions and it results in a shift in the sensor's response [8]. This shift is greater than the shift caused by the BGL variation. The findings will be presented in a future publication in more detail. However, the position can easily be controlled by placing the sensor in a housing that accommodates a finger. Such a housing was custom 3D printed and used as shown in Fig.5.

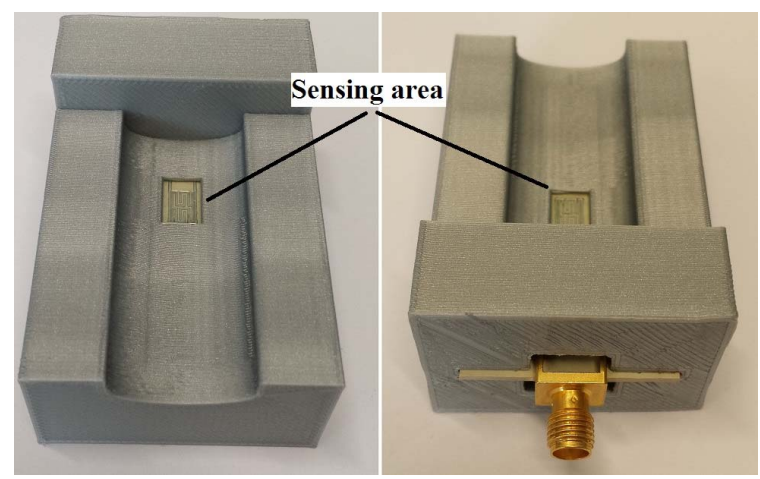

Fig. 5. 3D printed sensor housing 


\section{B. Pressure Applied onto the Sensor}

The sensor's frequency response highly depends on the pressure exerted by the fingertip onto the sensor. The effect of pressure was briefly discussed in [8], where a pressure sensor was used under the RF glucose sensor. Our experiments with our proposed sensor showed that a measurement with a fingertip is extremely sensitive to the pressure applied. The frequency difference in the resonant peaks between a light and a firm pressure on the sensor may be as high as $240 \mathrm{MHz}$ whereas the expected shift due to blood glucose variation is well under $10 \mathrm{MHz}$ [7]. This shows that if the pressure is not monitored precisely, the shift due to glucose variation will be masked under the shift resulting from the pressure variation causing measurement error.

However, the sensitivity to the pressure is expected for the following reasons:

- The effective thickness of the soft tissue layers change depending on the pressure applied

o The electrical coupling between the sensor and the fingertip varies depending on the pressure applied

o The blood is pushed away from the fingertip proportional to the pressure applied, altering the amount present for measurement

To the author's best knowledge, there is no work in the open literature discussing the importance of pressure applied onto the sensor or proposing a precision pressure sensing circuit. Using a pressure sensor such as a resistive force sensor under the RF glucose sensor does not provide the precision needed for repeatable results. For this reason, two novel pressure sensing circuits were proposed and are reported in this paper.

\section{The Proposed Novel Pressure Sensing Circuits}

The proposed circuit topology uses a 24-bit Capacitance to Digital Converter (CDC) chip and acts as an interface between the RF glucose sensor and the Vector Network Analyzer (VNA) utilizing the RF glucose sensor as a capacitive pressure sensor. The CDC uses a $16 \mathrm{kHz}$ excitation signal and the RF sensor provides a good sensitivity to proximity and pressure due to its highly capacitive nature. However, the pressure sensing circuit can be paired with any 1-port RF sensor.

Two variations of the topology are proposed. The first circuit (Ver.1) incorporates an RF switch between the RF sensor, CDC and the VNA. A Microcontroller Unit (MCU) is used to control the RF switch and the CDC, generate a trigger signal to trigger the VNA for a measurement and send pressure information to a computer. A target pressure value is preset in the MCU and the RF sensor is connected to the CDC via the RF switch. When the target pressure level is reached, the RF switch connects the RF sensor to the VNA and the MCU triggers the VNA for a measurement. The second circuit (Ver.2) allows for continuous measurement eliminating the RF switch and using a high-pass filter (HPF) on the VNA line to block the excitation signal in that branch and an RF choke (RFC) on the CDC line to block the RF signal in that branch.
The pressure data is continuously sent to the computer by the MCU. The circuit block diagrams are shown in Fig.6 for the proposed circuits.

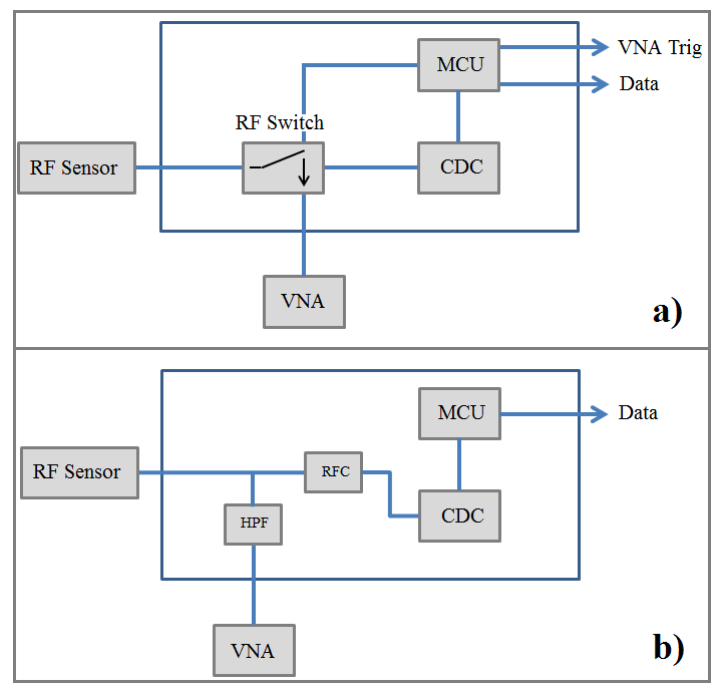

Fig. 6. Diagrams for: a) Circuit Ver.1 b) Circuit Ver.2

The photographs of the designed and realized circuits are shown in Fig. 7.

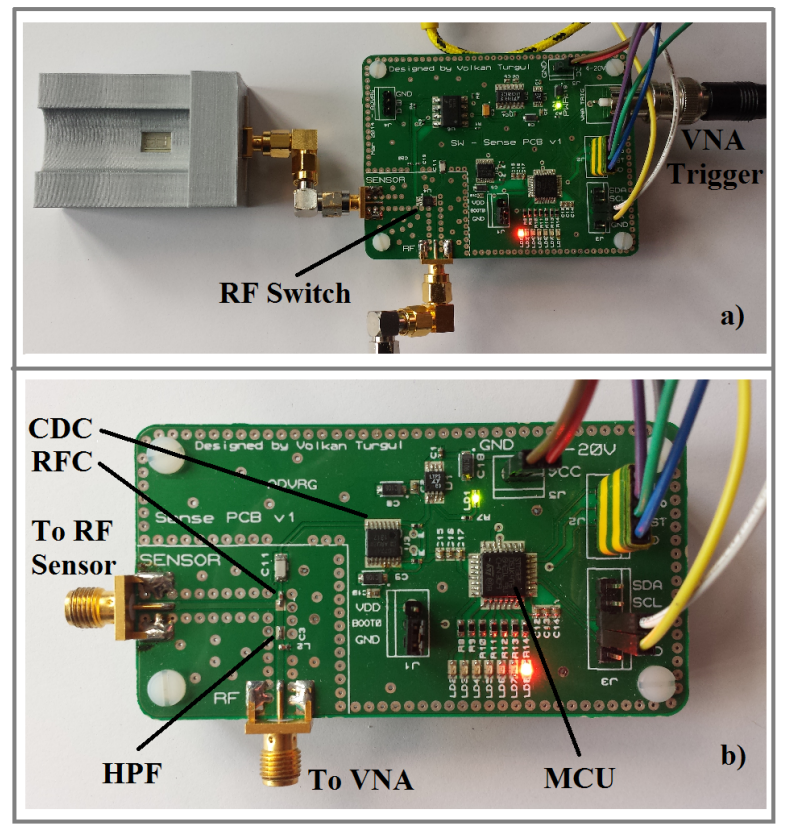

Fig. 7. Photographs of: a) Circuit Ver.1 b) Circuit Ver.2

Three experiments were carried out to evaluate the performance of the proposed circuits. First, a blind test was performed with the sensor. The sensor was connected to the VNA directly, a test subject was asked to place a finger on it and the resonant frequency was recorded. This was repeated 20 times re-placing the finger where the subject was asked to apply approximately the same pressure each time without knowing the actual sensor response to avoid positive reinforcement. The results are shown in Fig. 8. 


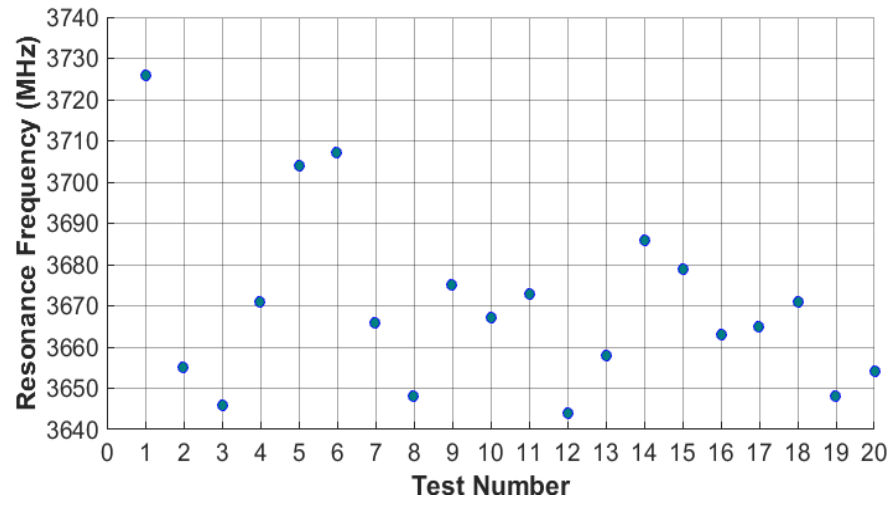

Fig. 8. Resonant frequency recorded in 20 tests (Sensor directly connected to the VNA)

It can be seen from the results that the resonant frequency varies substantially (standard deviation $=21.73 \mathrm{MHz}$ ) while trying to apply the same pressure each time.

Next, the circuit Ver.1 was tested the same way where a preset pressure value was programmed into the MCU. The test subject is guided on the pressure level via onboard LED lights and the VNA is triggered at the desired pressure level. The results for 20 measurements are shown in Fig. 9.

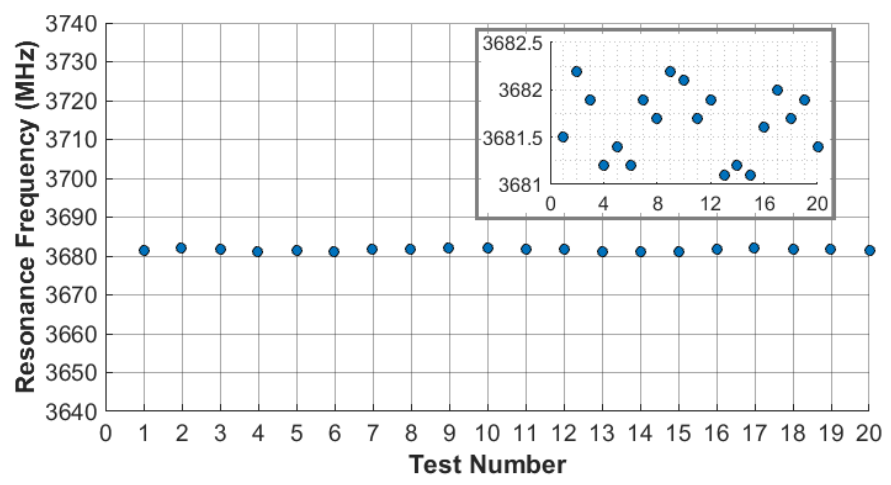

Fig. 9. Resonant frequency recorded in 20 tests (Sensor connected to the VNA through circuit Ver.1)

It can be clearly observed that the variation between measurements is very little with a standard deviation of $0.366 \mathrm{MHz}$, significantly improving the measurement accuracy. The accuracy can be further improved by averaging a number of measurements.

Finally, the relationship between the resonance frequency and the pressure applied was established using the circuit Ver.2. About 300 measurements were taken continuously while varying the pressure applied onto the RF sensor. Pressure was varied randomly between light and firm levels and result were plotted real-time in MATLAB with the frequency response gathered from the VNA and the pressure data received from the proposed circuit. The obtained graph is shown in Fig. 10. The graph shows that the resonance frequency is almost a linear function of the pressure applied and a good repeatability can be achieved for a specific pressure level.

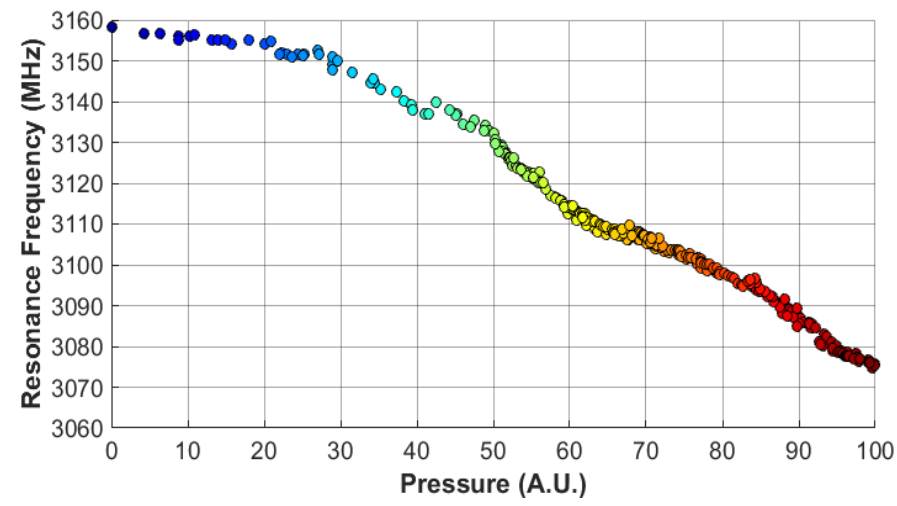

Fig. 10. Resonant frequency vs. pressure applied (Sensor connected to the VNA through circuit Ver.2)

\section{CONCLUSION}

The pressure applied onto the RF glucose sensor is critical to get repeatable results, independent of the type of sensor used. The effective thickness of the biological layers changes depending on the pressure applied; hence, the sensor's frequency response also varies accordingly. For this reason, the pressure needs to be accurately monitored while taking blood glucose readings with an RF sensor. However, conventional pressure sensors require a lot of force to be applied which results in a crude resolution and do not provide enough sensitivity. Therefore, the glucose measurement is hindered by the effects of inconsistent pressure applied. To solve this problem two novel pressure sensing circuits were proposed making use of the already existing RF glucose sensor a pressure sensor too. A good accuracy and repeatability were achieved as presented.

\section{REFERENCES}

[1] "Diabetes Atlas, 6th ed.," International Diabetes Federation (IDF), 2015. [Online]. [Accessed 05 2016].

[2] R. Holt, C. Cockram, A. Flyvbjerg and B. Goldstein, Textbook of Diabetes, New York, NY: John Wiley \& Sons, 2011.

[3] S. Vashist, "Continuous Glucose Monitoring Systems: A Review," Diagnostics, vol. 3, no. 4, pp. 385-412,, 2013.

[4] A. V. Vorst, A. Rosen and Y. Kotsuka, RF/microwave interaction with biological tissues, Hoboken, NJ: John Wiley \& Sons, 2006.

[5] K. Cole and R. Cole, "Dispersion and Absorption in Dielectrics I. Alternating Current Characteristics," The Journal of Chemical Physics, vol. 9, no. 4, p. 341, 1941.

[6] S. Gabriel, R. Lau and C. Gabriel, "The dielectric properties of biological tissues: III. Parametric models for the dielectric spectrum of tissues," Physics in Medicine and Biology, vol. 41, no. 11, pp. 2271-2293, 1996.

[7] V. Turgul and I. Kale, "Characterization of the Complex Permittivity of Glucose/Water Solutions for Noninvasive RF/Microwave Blood Glucose Sensing," in IEEE International Instrumentation and Measurment Technology Conference (I2MTC), Taipei, 2016.

[8] J. T. Bernhard and C. J. Tousignant, "Resonant frequencies of rectangular microstrip antennas with flush and spaced dielectric superstrates," IEEE Transactions on Antennas and Propagation, vol. 47, no. 2, 1999.

[9] B. Jean, E. Green and M. McClung, "A microwave frequency sensor for non-invasive blood-glucose measurement," in IEEE Sensors Applications Symposium, Atlanta, GA, 2008. 